\title{
DNA2App: Mobile sequence analyser
}

\author{
Jia-Zhi Sim ${ }^{1}$, Phi-Vu Nguyen ${ }^{1}$, Yu Zang ${ }^{1}$ and Samuel Ken-En Gan ${ }^{1,2^{*}}$
}

\begin{abstract}
Smartphones have changed the convenience of everyday life and promises to facilitate core biomedical research analysis. To materialize this for research, we created DNA2App, a free standalone native Android and iOS mobile application that analyzes nucleic acid and amino acid sequences. Boasting a built-in database of more than 240 restriction sites and the amino acid codon table, the app allows sequences to be reverse complemented, translated in all 3 frames and searched for restriction sites, and their GC content and melting point can be calculated. Supplemented by the features of exportation to FASTA format and copying to clipboard, results can be kept or easily stored and communicated. Combined, these features increase the ease and convenience of commonly used sequence analyses that every scientist can carry in their pockets.
\end{abstract}

\section{Introduction}

Sequence analyses of nucleic acid and proteins play a fundamental role in biomedical and clinical research. In the lab, almost all molecular biology experiments such as Polymerase Chain Reaction (PCR), recombinant gene manipulation and gene synthesis rely on such analyses. It is also through sequence analysis that diseases (e.g. sickle-cell anemia) are detected.

To increase the productivity of these experimental processes, the scientist would benefit from simple accessible apps that can give quick and crucial sequence analysis. This demand is highlighted in the numerous new biomedical apps that increase the portability and efficiency of biological research. Examples of apps that do this include DNAApp (Nguyen et al. 2014), GelApp (Sim et al. 2015), and YASARA View (Krieger \& Vriend 2014). These aid in everyday experiments (see lab examples (Poh \& Gan 2014a; Poh \& Gan 2014b)) to improve the presentation and accuracy of experimental data (see example (Ling et al. 2015)). One key feature of these apps is that they are not affected by internet connectivity which may otherwise limit analysis speeds and accessibility.

To address the need for quick, portable, and internetindependent sequence analysis, we developed DNA2App a mobile app that performs the commonly used analysis of nucleic acid and amino acid sequences. When used

\footnotetext{
* Correspondence: samuelg@bii.a-star.edu.sg

${ }^{1}$ Bioinformatics Institute, Agency for Science, Technology, and Research (A*STAR), Singapore 138671, Singapore

${ }^{2}$ p53 Laboratory, Agency for Science, Technology, and Research (A*STAR), Singapore 138648, Singapore
}

together with "app enablers" like DNAApp (Nguyen et al. 2014) which analyzes ab1 files, DNA2App can provide additional off-line analysis of sequencing data. While sharing certain similar features to our DNAApp, DNA2App was highly requested as it allows text input, and has added features (such as restriction enzymes, reverse translation) compared to only ab1 files analysis in DNAapp. Thus it serves as an independent app and also a complementary add-on for mobile analysis.

DNA2App was built with the following features (see Fig. 1): Reverse Translation from amino acid sequence to nucleic acid; Export to FASTA; Copy to clipboard; Nucleic acid sequence reverse complementation; Nucleic acid translation in all six frames (when used together with reverse complementation); Restriction site analysis; Target sequence search; GC content percentage; and melting temperature calculation $(\mathrm{Tm})$. To ensure functionality regardless of device internet connectivity, these features are all built into the app without the need for mobile network/WIFI or access to multiple websites.

DNA2App for Android was developed using version 4.4 of the Eclipse Integrated Development Environment, Luna and utilises the 'Simple File Dialog', developed by Scorch Works (http://www.scorchworks.com/Blog/simple-file-dialog-for-android-applications/). 'Simple File Dialog' license is under the Code Project Open License (CPOL) 1.02). The iOS version was developed using Objective-C using XCode 6.2.
Springer

(c) 2016 Sim et al. Open Access This article is distributed under the terms of the Creative Commons Attribution 4.0 International License (http://creativecommons.org/licenses/by/4.0/), which permits unrestricted use, distribution, and reproduction in any medium, provided you give appropriate credit to the original author(s) and the source, provide a link to the Creative Commons license, and indicate if changes were made. 


\section{Nucleotide/ amino acid sequence check}

For DNA analysis, DNA2App checks the entry to ensure only acceptable letter are entered (other letters are marked in different colors). These include A, T, C, and G for translation; additional $\mathrm{U}$ and $\mathrm{N}$ for reverse complementation; and single-letter amino acid letters together with "*" for stop codons. The window also allows the pasting of sequences copied from other apps, including web browsers or emails. To facilitate entries, we have only enabled certain letters in the nucleic acid panel, where only the acceptable letters can be registered for input.

\section{Restriction sites and sequence search}

Users can search for specific defined sequences or restriction sites from the database of more than 240 different enzymes (for nucleic acid sequences only). Segments where the sequence matches the desired search sequence will be shown in green fonts (see left panel of Fig. 1). With userdefined input, primer sequences and codons of interest can be identified easily.

\section{GC content $\%$ and melting temperature (Tm)}

In the nucleic acid analysis window, there are two buttons below the text field for GC content and the melting temperature $(\mathrm{Tm})$ calculations.

After checking for illegal characters, GC content calculates the total number of Guanine and Cytosine over the total number of bases and expresses it as a percentage.
For Tm calculation, the following formulae were used for sequences less than 14 nucleotides:

$$
\operatorname{Tm}=(A+T) * 2+(G+C) * 4
$$

For sequences with more than 13 nucleotides, the adjusted formulae is:

$$
\operatorname{Tm}=64.9+41 *(G+C-16.4) /(\mathrm{A}+\mathrm{T}+\mathrm{G}+\mathrm{C})
$$

The formulae were obtained from http://www.biophp.org $/$ minitools $/$ melting_temperature $/$ demo.php?formula= basic .

\section{Reverse complement}

DNA2App displays the reverse complement sequence output below the input sequence (see center panel of Fig. 1). Users can search, export to FASTA, and use the copy to clipboard for other analyses or delivery by emails/social media.

\section{Protein translation}

Nucleic acid sequences are translated to amino acids in all three reading frames. Combined with the reverse complement feature, DNA2App can provide all six reading frames (see right panel of Fig. 1). For easy reference, input sequence and the translation frames are displayed in a scrollable text box.

As with the other features, the copy to clipboard, export to FASTA and search functions can be used.

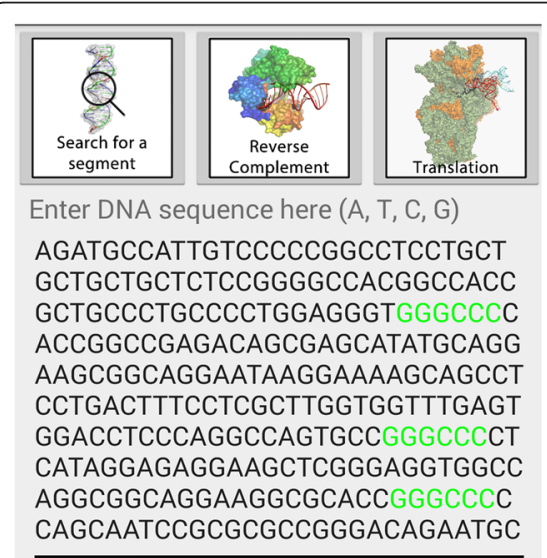

CAGCAATCCGCGCGCCGGGACAGAATGC

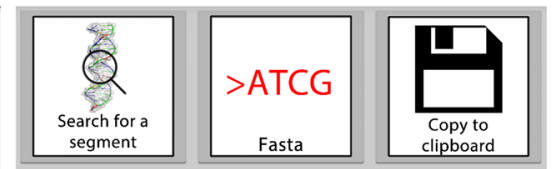

Reverse complemented sequence TTCAGGTCTGTAATTAAACTTGCGTGAG CATTCATGGGTGAGGTTTTATTTGCAGG AGGAGAAGGTCTTCCAGAAGAAGTTCC TGCAGGGCATTCTGTCCCGGCGCGCGG ATTGCTGGGGGCCCGGTGCGCCTTCCT GCCGCCTGGCCACCTCCCGAGCTTCCT CTCCTATGAGGGGCCCGGCACTGGCCT GGGAGGTCCACTCAAACCACCAAGCGA GGAAAGTCAGGAGGCTGCTTTTCCTTA TTCCTGCCGCTTCCTGCATATGCTCGCT

Original sequence:

AGATGCCATTGTCCCCCGGCCTCCTGCT GCTGCTGCTCTCCGGGGCCACGGCCAC CGCTGCCCTGCCCCTGGAGGGTGGGCC CCACCGGCCGAGACAGCGAGCATATGC AGGAAGCGGCAGGAATAAGGAAAAGC AGCCTCCTGACTTTCCTCGCTTGGTGGT TTGAGTGGACCTCCCAGGCCAGTGCCG GGCCCCTCATAGGAGAGGAAGCTCGGG AGGTGGCCAGGCGGCAGGAAGGCGCA CCGGGCCCCCAGCAATCCGCGCGCCGG

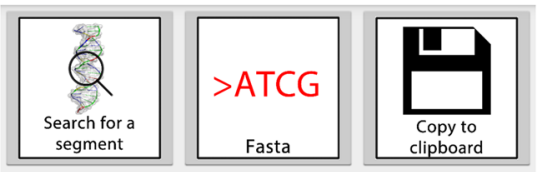

DNA to Protein Translation

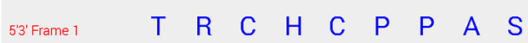
ACAAGATGCCATTGT CCCCCGGCCTCC Q $D$ A A I $V$ V $P$ P $R$ P $P$ P ACAAGAT GCCATT GT CCCCCGGCCT CC

K $M \quad M \quad P \quad L \quad S \quad P \quad G \quad L$ ACAAGATGCCATTGT CCCCCGGCCTCC

Fig. 1 DNA analysis features segment search (left), reverse complementation (middle) and protein translation in all 3-frames (right) 


\section{Reverse translation}

To determine the nucleic acid sequence from a protein sequence, DNA2App incorporated the 'Reverse Translation' feature. The most commonly used codons in Escherichia .coli (see www.bioinformatics.org/sms2/ rev_trans.html) were used.

For easy reference, the protein sequences will be shown above the nucleic acid sequence. Both the nucleic acid and amino acid sequence can be scanned through for specific sequences, copied to clipboard, and exported to FASTA format.

\section{Conclusion}

DNA2App is a standalone Android and iOS app that incorporates common molecular biology analyses such as restriction site search, reverse complementation, translation, reverse translation, as well as \% GC content and $\mathrm{Tm}$ calculations. Totally independent of the internet, the researcher has the convenience to do all the common analysis in one app without using multiple bioinformatics websites. Coupled with other apps, analysis of sequences (both protein and DNA) can now be more comprehensive and standalone on the smartphone.

\section{Availability and implementation}

DNA2App for Android and iOS are freely available on the Google Play Store and Apple App Store as 'DNA2App'. More details on the app can be found at www.facebook.com/APDLab; www.bii.a-star.edu.sg/research/trd/apd.php.

The 'DNA2App' user guide is available at http://tinyurl.com/DNA2App, and a video tutorial is also available on the Google Play Store.

DNA2App requires Android version 3 and above, and iOS 8.2 or later.

The "Export to FASTA" feature saves to the local drive on Android devices. For iOS devices, this feature saves to Dropbox as iOS security features prevent writing to local drives.

\section{Competing interests}

The authors declare that they have no competing interest.

\section{Authors' contributions}

JZ designed the layout and made the Android version of the app. PV provided the codes for translation and reverse translation features for both Android and iOS versions of the app. YZ made the iOS version. SKE conceived the idea, directed the design and implementation of the app. All authors read and approved the final manuscript.

\section{Acknowledgements}

This work is funded by JCO1334i00050 grant, from the Joint Council Office, Agency for Science, Technology, and Research, Singapore. We thank $\mathbb{H}$ Budianto and EHW Tan for their help in debugging.

Received: 4 January 2016 Accepted: 5 January 2016 Published online: 02 April 2016

\section{References}

Nguyen P-V, Verma CS, Gan SK-E. DNAApp: a mobile application for sequencing data analysis. Bioinformatics. 2014;30(22):3270-1. doi:10.1093/bioinformatics/btu525.

Sim J-Z, Nguyen P-V, Lee HK, Gan SK-E. "GelApp: Mobile gel electrophoresis analyser." Nat Methods. 2015; doi:10.1038/an9643.

Krieger E, Vriend G. YASARA View - molecular graphics for all devices - from smartphones to workstations. Bioinformatics. 2014;30(20):2981-2.

Poh JJ, Gan SKE. "The Determination of Factors Involved in Column-Based Nucleic Acid Extraction and Purification. J Bioprocess Biotech. 2014a;4:157. doi:10.4172/2155-9821.1000157.

Poh J-J, Gan SK-E. Comparison of customized spin-column and salt-precipitation finger-prick blood DNA extraction. Biosci reports. 2014b;34(5):629-34. doi:10.1042/BSR20140105.

Ling W-L, Lua W-H, Gan SK-E. "Fast reversible single-step method for enhanced band contrast of polyacrylamide gels for automated detection". Electrophoresis. 2015; doi: 10.1002/elps.201500094.

\section{Submit your manuscript to a SpringerOpen ${ }^{\circ}$ journal and benefit from:}

- Convenient online submission

- Rigorous peer review

- Immediate publication on acceptance

- Open access: articles freely available online

- High visibility within the field

- Retaining the copyright to your article

Submit your next manuscript at springeropen.com 